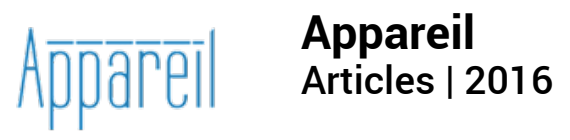

\title{
Benjamin et le paradoxe de la chaussette
}

\author{
Jean-Louis Déotte
}

\section{(2) OpenEdition \\ Journals}

\section{Electronic version}

URL: http://journals.openedition.org/appareil/2354

DOI: 10.4000/appareil.2354

ISSN: 2101-0714

\section{Publisher}

MSH Paris Nord

\section{Electronic reference}

Jean-Louis Déotte, « Benjamin et le paradoxe de la chaussette», Appareil [Online], Articles, Online since 07 November 2016, connection on 30 July 2020. URL : http://journals.openedition.org/appareil/2354 ; DOI : https://doi.org/10.4000/appareil.2354

This text was automatically generated on 30 July 2020 .

\section{(c) (i) (9)}

Appareil est mis à disposition selon les termes de la Licence Creative Commons Attribution - Pas d'Utilisation Commerciale - Pas de Modification 4.0 International. 


\title{
Benjamin et le paradoxe de la chaussette $^{1}$
}

\author{
Jean-Louis Déotte
}

À S. A.

\section{Sur une fréquentation discontinue}

1 Je me suis rendu compte avec le temps, depuis que je fréquente vraiment Benjamin, soit depuis la fin des années 1980 qui ont vu la publication des traductions de textes majeurs comme Le Livre des Passages, les Écrits français, que mon rapport à son œuvre était distrait (au sens benjaminien de Zerstreuung), c'est-à-dire non contemplatif, parce que sinon je m'y serais noyé comme le fameux peintre chinois de L'Euvre d'art à l'époque de sa reproductibilité technique où l'opposition perception de contemplation/perception de distraction est structurante. Une telle lecture est sensible aux éclats d'un texte, sans chercher à établir la vérité de l'œuvre (mais n'y a-t-il pas en général pour un écrit que des lectures? Une dissémination de lectures ?), car elle rompt avec le programme qui avait été celui de Benjamin pour rendre compte de son interprétation des Affinités électives de Goethe, son premier grand texte critique (le schéma de l'œuvre palimpseste, l'opposition entre le noyau de vérité et la teneur chosale, c'est-à-dire son contexte). Comme le second Benjamin, celui qui suit la découverte bouleversante pour lui de Giedion $^{2}$, déclare ne pas citer des textes à interpréter, mais seulement les exposer, comme on le fait d'objets, j'ai suivi ce principe. Ce qui revient d'un côté à isoler ses fameuses citations sans guillemets, c'est-à-dire ses archives qui n'ont pas besoin d'être interprétées, qu'il faut prendre littéralement, comme les documents chez Foucault, de l'autre à structurer le texte sous la forme de rapports, de rapports de rapports.

Quand les experts de l'École de Francfort déclarent que Benjamin n'est pas traduisible, et reste incompréhensible pour les Français, malgré ce que lui-même écrit sur la traduction ( $L a$ Tâche du traducteur: le «bon» traducteur élabore des fragments de la langue de vérité), cela revient à dire au fond que ses textes sont inintelligibles pour 
tout le monde. Au contraire, si l'on cherche à retrouver l'armature (oserais-je écrire technique?) de sa pensée, selon le principe de la quatrième proportionnelle, connaissant trois des termes mis en rapports, il est aisé de caractériser le quatrième. Ainsi quand il compare le cinéaste, le peintre, le chirurgien et le chaman du point de vue de la pénétration de la chose (le corps, l'œuvre peinte), sachant que le peintre focalisant sur son œuvre manque de s'y perdre (rapport de contemplation), que le chirurgien pénètre avec ses instruments le corps du patient alors que le chaman se borne à le surplomber, on peut déduire que le cameraman pénètre les chairs du collectif ${ }^{3}$, et que l'appareil qu'il utilise pour cela doit disparaître au moment de la projection du film. Une autre analogie confirme cette déduction: la caméra donne accès à l'inconscient optique de la même manière que la psychanalyse livre l'inconscient psychique. Mais comme il s'agit des chairs du collectif, on peut préciser que l'objet du cameraman, ce n'est pas tant le monde onirique en tant que tel (Kracauer précisera ce point dans sa Théorie du film), mais le lieu commun par excellence: le substrat physiologique de la rêverie collective, le kinesthésique, un en deçà des affects. Le geste technique du dessinateur et du cameraman est pensé alors comme innervation du corps singulier ou collectif, dans la continuité de Fiedler, du premier Freud et des Surréalistes. C'est une émancipation (prothétique) ou une révolution (politique).

On peut continuer notre meccano avec d'autres mises en rapports : voulant lancer un curieux défi à Giedion, il déclare vouloir le dépasser et là où l'historien de l'architecture a pensé les rapports de l'architecture pré-moderne en termes d'infrastructure versus superstructure (construction technique versus façade académique), Benjamin propose plutôt le rapport suivant : processus physiologique versus rêve. Ceci est, à première vue, tout à fait déconcertant, et on comprend que les « experts " préfèrent ne pas avoir à traduire. La méthode que j'ai pratiquée a consisté à postuler que chaque fragment du texte benjaminien était structuré séquence après séquence, et que l'on devait pouvoir reconstruire la structure profonde, l'armature de sa démarche de pensée. Dès lors, une séquence grammaticalement obscure pourra être mise en rapport avec une autre structure séquentielle qui lui donnera sens. C'est le cas de la Thèse VIII de l'ouvrage Sur le concept d'histoire: la question de l'état d'exception s'entend à partir de l'interprétation de l'événement déjà vu. Il faut ici mobiliser une importante thématique, celle du déjà-vu, ce que j'ai commencé à faire dans Qu'est-ce qu'un appareil ?4 en insistant sur le fait, comme le montre Remo Bodei ${ }^{5}$, que si l'humanité a toujours perçu le déjà-vu, il revient au XIX $x^{e}$ siècle de l'avoir conceptualisé. C'est donc un trait d'époque, caractérisant la contemporanéité des singularités vivant dans ce siècle.

4 Certes, les façades du XIX siècle, en particulier celles des gigantesques galeries d'exposition, comme le Grand Palais à Paris, n'étaient pas cohérentes avec leur infrastructure industrielle parce qu'elles relevaient du rêve collectif, passéiste et patrimonial de l'époque (autre facteur de contemporanéité). Mais alors, logiquement, le rêve tel que veut le penser Benjamin est l'expression collective des processus physiologiques de la foule en mouvement dans ces galeries. Dès lors, Benjamin a trouvé son angle d'attaque et son objet : le mouvement de la masse, comme s'il se produisait au fond d'un aquarium qu'on appelle l'atmosphère. Cette conception du mouvement et la fantasmagorie propre au passage urbain comme monde liquide proviennent du Paysan de Paris d'Aragon. Nous n'avons donc pas, comme chez les marxistes orthodoxes, les rapports de production versus l'idéologie, mais une sorte de physio-psychologie des profondeurs (les rêveries collectives de ce siècle), pas très éloignée de celle de Jung, mais ne flirtant pas avec le caractère destinal des mythes originaires. Reste que pour 
Benjamin, le XIX ${ }^{e}$ siècle entretient avec l'originaire, c'est-à-dire avec la "préhistoire " (Urgeschichte), un rapport de grande proximité, un rapport exemplaire, car alors la fabrique du rêve collectif (la fantasmagorie originaire) fut plus tangible que jamais. Si les processus physiologiques sont identiques pour tout le genre humain, il n'en va pas de même pour les rêves qui les ont exprimés (les fantasmagories secondaires, historiquement déterminées). C'est que le XIX siècle a été généré par d'autres opérateurs de fantasmagorie que les siècles précédents. Ces opérateurs historiques médiatisent les rapports: processus physiologique versus rêves. Le passage urbain est l'un de ces opérateurs. C'est dans cet esprit qu'il abordera, pour le $\mathrm{xx}^{\mathrm{e}}$ siècle, l'étude sur l'appareil cinématographique. L'un et l'autre sont des appareils immersifs, même si par ailleurs l'appareil cinématographique appartient aussi à la série projective (cf. plus bas). Car l'un et l'autre configurent la sensibilité de la masse qui, dans le même temps, les absorbe selon une logique de la double inclusion. J'ai donc insisté sur cette fabrique de l'urbanité par l'un et l'autre, en insistant sur la production d'une spatialité nouvelle : l'immersion. C'est la clef de ce qu'on appellera "psychologie des foules », préalable à l'étude des mouvements totalitaires. Dans ce cadre, des notions philosophiques et politiques comme celles de "prise de conscience», de "critique de la culture», de « subjectivation », etc., n'ont plus de sens.

5 Je laisse de côté l'opposition entre aura et trace, que j'ai analysée ailleurs ${ }^{6}$ et qui serait à reconsidérer du point de vue de l'éloignement versus la proximité. Le chaman et le peintre classique sont du côté de l'aura, le cameraman et le chirurgien de la trace.

Dès lors, si Benjamin est un penseur du politique, et pour considérer quelle politique fut la sienne, il faut au préalable reconstituer l'architectonique de l'action. La question du lieu de l'action devient alors incontournable. Aussi faut-il considérer, à la suite de Simmel par exemple, la question du logement dans ses rapports avec l'espace commun, parce que là aussi le $\mathrm{XIX}^{\mathrm{e}}$ siècle est au plus près de l'originaire: le logement du " particulier » cher à Louis-Philippe est un étui. Or l'étui est l'enveloppe première de l'humain. Bien avant Henri Lefebvre, qui connaissait peu Benjamin, ce dernier ne pensa pas l'action politique en dehors de la socio-politique de l'espace ${ }^{7}$.

\section{Critique de la subjectivation}

7 L'étude sur Descartes, et en particulier celle des deux premières Méditations métaphysiques, m'a permis de montrer que la structure du cogito, soit la coïncidence instantanée de la pensée et de l'existence, reprenait sur un plan philosophique la structure de la démonstration de la perspective par Brunelleschi, à partir de l'analyse de Damisch$^{9}$ (la coïncidence du point de vue et du point de fuite).

8 Or, si comme l'écrit Hegel, la vérité a dès lors reçu une nouvelle assiette (l'évidence), l'instantanéité de ce moment essentiel doit être confirmée par une réalité qui ne disparaîtra pas l'instant d'après. D'où la nécessité de la représentation, laquelle n'est possible que grâce à la production d'appareils projectifs, notamment du premier d'entre eux: l'appareil perspectif. C'est la raison, me semble-t-il, de l'immense production picturale et sculpturale qui va accompagner la Renaissance italienne ${ }^{10}$.

9 J'ai esquissé, à partir de là, la succession des appareils qui avaient en commun une base identique: l'écriture projective (voir plus bas). De ce point de vue, l'appareil cinématographique réalise la synthèse (et l'analyse) de ces appareils projectifs (camera 
obscura, perspective, musée, photographie, psychanalyse) dans la mesure où il consiste en un montage-démontage d'appareils. C'est Benjamin qui a le mieux compris cet aspect essentiel :

Le cinéma: démêlement (résultat?) de toutes les formes de vision, de tous les rythmes et de tous les temps préformés dans les machines actuelles, de telle sorte que tous les problèmes de l'art actuel ne peuvent trouver leur formulation

définitive qu'en corrélation avec le film ${ }^{11}$.

10 Si tous ces appareils parlent la même « langue " parce qu'ils sont tous projectifs, alors l'action politique sera d'essence représentative, à la différence de l'action politique dans les démocraties grecques ou dans la république romaine. C'est la difficulté centrale que rencontre Machiavel dans son Discours sur la Première décade de Tite Live.

\section{La fantasmagorie n'est pas l'idéologie}

11 C'est parce que Benjamin n'est pas marxiste (car, en vérité, qu'a-t-il lu de Marx ?), que l'on peut suggérer qu'il développe une théorie de la culture bien différente. C'est ce que j'avais découvert dans sa lecture et sa présentation de l'œuvre d'E. Fuchs, collectionneur et historien ${ }^{12}$. Dans cette étude, il se livre à une critique de la conception social-démocrate de l'histoire, étude qu'il parachèvera dans les Thèses. Mais je pensais encore à cette époque qu'il fallait l'aborder par la «philosophie » de l'histoire, comme le faisait Françoise Proust ${ }^{13}$. Une expression résumait sa pensée : l'histoire peut se retourner comme un gant. J'en concluais à la réversibilité ${ }^{14}$ des enchaînements historiques, au "messianisme sans messie» selon Derrida, à l'irruption de l'improbable, soit le contraire de l'« État d'exception » de Schmitt. Or, depuis, j'ai appris à lire Benjamin en respectant la lettre de ses expressions, sage précepte élaboré par Berman $^{15}$ à propos de sa conception de la traduction.

On peut, à partir de là, tirer deux conséquences, dont la portée est fondamentale :

13 1) Si l'on prend l'expression au pied de la lettre, l'essentiel n'est plus la théorie de l'événement, mais l'espace du gant. L'histoire s'enroule et se déroule, fait des plis et des déplis, comme la fameuse chaussette d'Enfance berlinoise (Armoires) dont Benjamin explore les états : en boule, sous la forme d'une bourse, c'est la forme; déployée, prête à l'usage, c'est le contenu (la matière), et ce qui réunit à l'identique ces deux états physiques : c'est le nom chaussette.

14 Nous sommes à l'évidence dans un espace topologique, comme l'est la bouteille de Klein. Dès lors, les métamorphoses que connaît la chose font disparaitre la distinction forme/contenu ou forme/matière. Ce sera encore plus évident à l'époque de ce que Lyotard appelait les Immatériaux, quand forme et matériaux sont de même " nature »: le langage algorithmique.

2) D'autre part, une conception renversante de l'histoire. L'exemple fameux est celui d'un genre artistique (Origine du drame baroque), ce qu'il appelle par ailleurs status : la réalité culturelle par excellence est prise dans le flux du devenir, mais parce qu'elle s'enroule perpétuellement sur elle-même comme un tourbillon, persiste en n'étant pas entraînée par le courant. Benjamin résout la contradiction : certes, écrit-il, il y a bien une histoire socio-politique dans laquelle les hommes sont entraînés, mais il y a aussi des genres et des appareils qui coexistent et persistent dans l'être depuis leur surgissement. Ce qui permet d'affirmer que, genres et appareils, ont pu surgir successivement à tel ou tel moment du devenir, mais une fois qu'ils ont été mis à flot, 
ils demeurent dans la coexistence. Dans ce monde du développement-enveloppement, un appareil ancien peut toujours en englober un plus récent, ce qui complique évidemment la question de la contemporanéité...

Une telle conception, topologique, de la culture ne peut avoir de sens dans le cadre d'une problématique hégéliano-marxiste de l'histoire et donc de la dialectique. Il faudra reprendre à nouveaux frais l'usage benjaminien de la notion de dialectique (" image dialectique », « dialectique au repos », etc.), car le milieu de la culture, c'est la fantasmagorie, c'est-à-dire un « espace » commun qui a la consistance de l'image et non celle de l'ordre des discours et des énoncés.

\section{L'appareillage de la fantasmagorie}

Les appareils ${ }^{16}$, appelons-les «modernes », que j'ai étudiés en particulier dans L'Époque des appareils, de la camera obscura arabe à la vidéo, ont en commun d'avoir une base d'écriture: l'écriture projective. Depuis ses débuts au xve siècle, à Florence, du fait d'architectes et de peintres qui avaient des compétences universelles (cf. Léonard), l'écriture projective s'est imposée, mondialisée et a renouvelé la science (Galilée, Newton, etc.). Sa caractéristique essentielle est la suivante : la surface d'inscription des signes, et en art le support du dessin est topologique au sens où entre les signes (ou traces) et la chose supposée désignée, il existe un rapport de continuité et de transformation. Ainsi, l'ovale que je dessine sur le plan transparent du tableau peut-il désigner une chose ronde que je vois là-bas, à travers le plan qui est comme une vitre. C'est la raison pour laquelle, la relation entre le signe et la chose est dite en art « imitative » ou "représentative ». L'esthétique qui s'est imposée et qui a régné dans les Académies était donc mimétique, et ce jusqu'aux avant-gardes. Mais il y a bien des manières de représenter, parce qu'il y a différents appareils projectifs et même au sein de l'appareil perspectif, plusieurs manières de construire le géométral.

Jonathan Crary ${ }^{17}$ décrit le stéréoscope, fort en usage au xix ${ }^{e}$ siècle, comme un appareil permettant grâce à deux miroirs orthogonaux de voir deux images légèrement dissemblables du même objet. Le peintre Courbet aurait pu l'utiliser pour certaines toiles, dont la fameuse Rencontre, ce qui permettrait de rendre compte de la frontalité de ses peintures et d'une organisation en espaces disjoints. Autre exemple: l'axonométrie est projective, mais ignore le point de fuite. Donc, elle ne privilégie plus le subjectivisme inhérent à la perspective classique. Or, le monde architectural contemporain à Benjamin, et qu'il aimait, c'est celui qui privilégie l'axonométrie : les maisons blanches d'Ibiza, Le Corbusier, le Bauhaus. C'est une des raisons de son refus de la notion de sujet, et une de celles qui font que, pour nous, le sujet ne peut être un acteur politique.

Actuellement, nous entrons très rapidement dans une nouvelle ère de l'écriture, non plus projective, mais numérique, algorithmique. En apparence, les résultats sont les mêmes: 'les «anciens" appareils projectifs ont été absorbés, miniaturisés, complexifiés, reconfigurés et synthétisés. Nous allons vers un appareil unique qui travaillera indifféremment avec les sons, les images, les textes, etc., réduits à des data : la discrétisation. Une image numérique n'est rien d'autre qu'un très long " texte », une suite d'énoncés invocalisables et qui n'appartiennent pas au visible. Dans un algorithme, on ne peut retrouver la distinction fondamentale, établie par Frege, entre Sinn et Bedeutung. Car la dimension (horizontale) du sens y est inexistante (c'est une 
succession de lettres et de symboles qui n'ont aucun sens) et la dimension "verticale » de la dénotation est absente. Il faudrait plutôt parler d'une dimension " pragmatique ", car c'est un programme d'action, ou de genèse, pour une machine. Effectivement, on entre alors dans le monde du pouvoir sur les êtres et les choses. Il n'y a donc plus de continuité, et donc plus de rapport topologique, entre ce que je vois sur l'écran numérique et la supposée chose qui sert de référence, car entre eux, il y a ces pages de programmes, ces algorithmes. Ainsi, le modèle explicatif et productif de la photographie n'est plus l'optique et la chimie, mais un processus technique qui s'apparente plus au $\operatorname{radar}^{18}$. Le passage de la photo argentique à la photo numérique marque la disparition de la photo comme appareil au profit de la photo comme média de communication. Il n'est même pas évident que l'on puisse encore parler de traces s'inscrivant sur un support : dès lors la notion d'appareil est peut-être dépassée ${ }^{19}$. projective, on pouvait faire des projets, car les dessins sur une feuille étaient aussi des desseins : les traits annonçaient un monde, peut-être inexistant, mais possible. Il y avait une différence ontologique entre l'être et le devoir-être (les idéalités kantiennes), ce qui permettait de fonder l'éthique. Il y avait des utopies. Or, rien de tel déjà chez Benjamin : c'est l'indice que l'on est en train de passer d'une ère de l'écriture à une autre. Dans son monde de la fantasmagorie, il n'y a pas de place pour l'utopie, sinon au passé, sur le modèle de la temporalité photographique.

21 C'est alors la trace, si l'on peut encore parler de trace, qui a changé de nature : elle est devenue un datum, c'est-à-dire une information que l'on a achetée à crédit pour agir immédiatement. Notre rapport au temps est sous la détermination de l'effectivité : un programme est écrit pour générer sans autre médiation que les ordinateurs, logiciels, langages, interfaces nécessaires, un monde enrichi de tout l'apport des techno-sciences. Un monde où la distinction entre forme et matière ne sera plus pertinente puisque les matériaux eux-mêmes seront de part en part artificiels, générés par du code, ce que Lyotard appelait les Immatériaux. Quand les architectes contemporains" décrivent les productions de cette architecture "non-standard ", ils baignent dans une nouvelle et véritable fantasmagorie. Celle d'un monde où la nature, étant de part en part connue, n'apporte plus de limites et où il n'y a plus de lutte des classes entre les architectesprogrammateurs et les ouvriers du chantier... Dès lors, j'opposerai projet et programme, idéalité et effectivité. L'idéalité d'une idéologie politique, toujours inachevée, et le programme d'un parti totalitaire, sans restes. Nous sommes, me semble-t-il, les contemporains de ces derniers.

\section{Être contemporains?}

Si un appareil est ce qui configure la sensibilité commune (c'est la première thèse de L'Cuvre d'art... de Benjamin, à propos des époques de la sensibilité où il est question de l'appareillage cinématographique et de son impact sur l'époque), on peut conclure qu'il fait monde comme le musée l'avait fait pour le XIX $x^{e}$ siècle (Benjamin citant Giedion) ou le baroque pour le XVII siècle (cf. le château de Versailles selon ce même Giedion). Ce qui entraîne à chaque fois une autre définition de la temporalité.

C'est la raison pour laquelle nous sommes des contemporains, dans une passivité immédiate. Quand Agamben ${ }^{20}$ réduit la contemporanéité des singularités à la ressemblance des rapports critiques que ces dernières entretiennent avec leur présent, 
il confond contemporanéité et simultanéité, et ce d'une manière parfaitement élitiste, parce que la critique est plutôt l'affaire des clercs. Si nous n'avons pas, de fait, choisi d'être des contemporains, nous pouvons agir dans la simultanéité, sur la même scène politique, dans l'entr-expression des singularités, nous pouvons choisir d'intervenir dans le même temps sur un même objet. Et ce faisant, nous pouvons constituer ce que Nancy, dans la continuation d'Arendt, appelle communauté.

Dès lors, la politique n'a de sens qu'au milieu des appareils, parce que les appareils en constituent le milieu. Mais quelles sont les conditions de notre présent, ce que Benjamin appelait «Maintenant de la connaissabilité »? Qu'attendaient BenjaminKracauer d'un cinéma, dont nous serions immanquablement les contemporains, pour qu'il n'appartienne pas à l'esthétisation du politique, un cinéma qui au fond ne consisterait que dans l'exposition pornographique des processus kinesthésiques, une sorte de fascisme de l'organique? L'étrangéisation de la réalité visible (terme que Kracauer reprend à Brecht), c'est-à-dire pour reprendre l'exemple proustien de la grand-mère de l'auteur saisie comme par effraction dans son intimité, comme par une photographie, une sorte d'arrêt sur l'image révélant la réalité plus que ne le ferait la simple perception vécue ou le souvenir lui-même ${ }^{21}$. Dans le cas de Proust : la très vieille femme qu'elle était devenue alors qu'il conservait en mémoire l'image affectueuse d'une tout autre personne. C'est un exemple cardinal pour Kracauer qui y revient plusieurs fois, même dans son ouvrage sur l'histoire.

Or, ce qui nous entraîne tous aujourd'hui, ce qui nous destine au sens fort, qui nous oblige comme une loi éthique, beaucoup plus que n'aurait pu le faire tel ou tel appareil du passé, cinéma compris, c'est le despotisme de la communication: ainsi les photographies numériques ne sont-elles plus destinées, après encadrement, à commémorer le souvenir des êtres chers, mais à circuler indéfiniment sur internet. La question de savoir si ces images sont indicielles ou iconiques ne se pose plus guère, tant elles sont prises dans des flux qui n'offrent d'autres prises que celles, fugaces, de l'immersion.

La plus petite manifestation publique est aujourd'hui surexposée par la multiplication des prises de vues par téléphone cellulaire : c'est notre contemporanéité. S'il y a une émeute, au lieu de voir des poings levés, des portables sont dressés pour communiquer l'événement, tout en adressant le message suivant à la communauté virtuelle: «j'y étais ». Il y a donc là une indéniable simultanéité : la conjugaison de l'être ensemble et du même moment. Un appareil photo, c'est déjà aujourd'hui un petit terminal de communication audio-vidéo. Les analyses qui permirent à Benjamin de montrer la possibilité d'un renversement entre être testé (par exemple par la caméra) et le retournement volontaire du test sur soi-même pour livrer à la multitude les meilleures apparences de soi-même (politisation de l'esthétique), ne donnèrent lieu qu'à des expérimentations marginales (cf. durant l'après-guerre, le Groupe Medvedkine). Or, il faut bien admettre qu'avec l'introduction de la 3D, les films deviennent de plus en plus spectraux (cf. W. Wenders: En mémoire de Pina Bausch) et donnent ainsi raison à Derrida $^{22}$. La question devient alors, et elle est politique et esthétique : peut-il y avoir une étrangéisation de la spectralité générale? 


\section{BIBLIOGRAPHY}

Agamben, G., Qu'est-ce que le contemporain ?, Paris, Rivages, 2008.

Benjamin, W., L'CEuvre d'art à l'époque de sa reproductibilité technique, Écrits français, éd. J.-

M. Monnoyer, Paris, Gallimard, 1991.

Benjamin, W., Paris, capitale du XIX ${ }^{e}$ siècle, Paris, Le Cerf, 1989. Trad. fr. Lacoste.

Berman, A., L'Age de la traduction. Commentaire de La tâche du traducteur de Benjamin, Saint Denis, PUV, 2008.

Bodei, R., La Sensation de déjà-vu, Paris, Le Seuil, 2007.

Crary, J., L'Art de l'observateur. Vision et modernité au XIX' siècle, Nimes, éditions J. Chambon, 1994.

Trad. fr. 1994.

Damisch, H., L'Origine de la perspective, Paris, Flammarion, 1993.

Déotte, J.-L., Qu'est-ce qu'un appareil ? Benjamin, Rancière, Lyotard. Paris, L'Harmattan, 2007.

- L’Homme de verre. Esthétiques benjaminiennes, Paris, L'Harmattan, 1999.

- De la compénétration des espaces chez W.Benjamin aux Unités d'habitation de Le Corbusier Revue

<appareil.revues.org> $\mathrm{n}^{\circ}$ 12, 2013.

- L'Epoque de l'appareil perspectif, Paris, 2001.

Derrida, J., Entretien avec Les Cahiers du cinéma : Le cinéma et ses fantômes, n 534, Paris, 1999.

Giedion, S., Construire en France, construire en fer, en béton, Paris. Editions de La Villette, éd.

allemande, 1928, trad. fr. 2000.

Kracauer, S., L'Approche photographique, in Sur le seuil du temps. Essais sur la photographie, Montréal, Presses de l'Université de Montréal, col. «Philia », 2013 ; Paris, 2014.

Porchet, M., La production industrielle de l'image, Paris, L'Harmattan, 2002.

Proust, F., L'histoire à contretemps, Paris, Le Cerf, 1994.

\section{NOTES}

1. Réponses à quelques objections après la publication de mon ouvrage, W. Benjamin et la forme plastique. Architecture, technique, lieux, Paris, L'Harmattan, 2012.

2. Giedion, S., Construire en France, construire en fer, en béton, éd. Allemande, 1928, trad. fr. 2000, Paris.

3. Benjamin, W, Thèse XIV de L'Euvre d'art à l'époque de sa reproductibilité technique, Écrits français, J.- M. Monnoyer (dir.), Paris, Gallimard, 1991.

4. Déotte, J. L., Qu'est-ce qu'un appareil ? Benjamin, Rancière, Lyotard. Paris, L'Harmattan, 2007.

5. Bodei, R., La Sensation de déjà vu, Paris, Seuil, 2007.

6. Qu'est-ce qu'un appareil?, op.cit., L'opposition aura/trace est dépassée avec le règne architectural du verre comme mode de compénétration des espaces. Parce que le verre ne permet pas l'inscription de traces et qu'il " est sans aura ». Le verre nous fait pénétrer dans le monde de la cendre, par-delà l'opposition symbole/allégorie (ruine). 
7. Déotte, J.L., De la compénétration des espaces chez W. Benjamin aux Unités d'habitation de Le Corbusier Revue <appareil.revues.org> $\mathrm{n}^{\circ}$ 12, 2013.

8. Déotte, J.L., L'Époque de l'appareil perspectif, Paris, L'Harmattan, 2001.

9. Damisch, H., L'Origine de la perspective, Paris, Flammarion, 1993.

10. Déotte, J. L. : L'Epoque de l'appareil perspectif. Brunelleschi, Descartes, Machiavel. Paris, L'Harmattan, 2001.

11. Benjamin, W. : Paris, capitale du XIXème siècle, p.412, Paris, Le Cerf, 1989, trad . fr. J.Lacoste..

12. Déotte, J.L. : L'Homme de verre. Esthétiques benjaminiennes, Paris, L'Harmattan, 1999.

13. Proust, F. : L'histoire à contretemps, Paris, Le Cerf, 1994.

14. Et en ce qui concerne l'espace urbain, comme Naples (A. Lacis, W. Benjamin : Journal de Naples), je concluais à la porosité. La réversibilité ou porosité pourraient être le maître mot de la pensée de Benjamin :

_ réversibilité entre la forme et la matière par l'évocation de l'une par l'autre ;

_ réversibilité entre le medium et le message, par exemple entre le livre et le texte. La sacralité du texte (cf. Thorah) entraîne celle du livre dans ses éléments les plus matériels (typographie, reliure, etc.). Il faudrait faire une recension des matériaux privilégiés pour l'entrecroisement, la compénétration comme la lave ou le verre. Pour la psyché, individuelle et collective, c'est évidemment le rêve. Tout ce qui permet le passage d'un état à l'autre.

15. Berman, A., L'Age de la traduction. Commentaire de La tâche du traducteur de W. Benjamin, Saint Denis, PUV, 2008.

16. Les notions d' "appareil " et d'appareillage sont largement utilisées par Benjamin dans L'œuvre d'art, mais sans être conceptualisées.

17. Crary, J., L'Art de l'observateur. Vision et modernité au XIXe siècle, Nimes, éditions J. Chambon, 1994. Trad. fr. 1994.

18. Porchet, M., La Production industrielle de l'image, Paris, L'Harmattan, 2002.

19. Revue en ligne <appareil.revues.org> $n^{\circ} 11,2013$; https://appareil.revues.org/1747

20. Agamben, G., Qu'est-ce que le contemporain ? , Paris, Rivages, 2008.

21. Kracauer, S., L'Approche photographique, in, Sur le seuil du temps. Essais sur la photographie. Trad. fr (Canada), Montréal, 2013. Paris, 2014.

22. Derrida, J., Entretien avec Les Cahiers du cinéma : Le cinéma et ses fantômes, nº 534, Paris, 1999.

\section{AUTHOR}

\section{JEAN-LOUIS DÉOTTE}

Jean-Louis Déotte est professeur émérite des Universités, philosophie, responsable de la collection Esthétiques chez L'Harmattan et co-responsable du thème 4 de l'axe 1 de la MSH Paris Nord. 